\title{
Flow of Information in Molecular Biological Mechanisms
}

\section{Lindley Darden}

Committee for Philosophy and the Sciences

Department of Philosophy

University of Maryland

College Park, MD USA

darden@umd.edu

\begin{abstract}
In 1958, Francis Crick distinguished the flow of information from the flow of matter and the flow of energy in the mechanism of protein synthesis. Crick's claims about information flow and coding in molecular biology are viewed from the perspective of a new characterization of mechanisms (Machamer, Darden, and Craver 2000) and from the perspective of information as holding a key to distinguishing work in molecular biology from that of biochemistry in the 1950s-1970s (Darden and Craver 2002). Flow of matter from beginning to end does not occur in the protein synthesis mechanism; flow of information (from Crick's perspective) does. The flow of information and coding in molecular biological mechanisms are distinguished, on the one hand, from formal information theory, and, on the other, from information as used in cognitive neuroscience, where information and representation are often coupled.
\end{abstract}

\section{Keywords}

genetic code, Francis Crick, information, mechanism, molecular biology, protein synthesis 
The term "information" was used by the molecular biologist Francis Crick in 1958 in stating hypotheses about the relation of genes to proteins, namely, that the linear order of bases in DNA determined the corresponding linear order of amino acids in proteins. As characterized by Crick, information was not static in the way that, say, coded words on a page are static. Instead, Crick's characterization of information, as we will see, was dynamic, that is, it required a mechanism operating to carry out a task. Crick also distinguished three different kinds of flows in the mechanism of protein synthesis: flow of information, flow of matter, and flow of energy. As a molecular biologist, Crick explicitly focused his attention on flow of information, and not on flow of matter or flow of energy. He discussed biochemical work dealing with matter and energy flow.

Crick's work serves as the basis for two claims, one philosophical, the other historical. First, "information" as used in molecular biology requires the operation of a mechanism. The characterization of mechanism in previous work (Machamer, Darden, and Craver 2000) aids in seeing the role of a mechanism in Crick's use of "information." Discussion of mechanisms also aids in formulating the as-yet-unanswered question of the difference between mechanisms in which information may be said to flow and mechanisms where no information flow is said to occur. Second, the idea of molecular biology as studying the flow of genetic information aids in distinguishing molecular biology from biochemistry in the 1950s and 1960s. Early biochemical work on protein synthesis investigated flow of matter and flow of energy, not flow of information (Darden and Craver 2002). Biochemistry, prior to its interfield interaction with molecular biology, lacked the idea of information flow from the genetic material of DNA to the ordering of amino acids in proteins. Most biochemical work centered on chemical reactions catalyzed by enzymes, in which information flow was not found.

\section{Crick on Information}

Crick's 1958 paper, "On Protein Synthesis," is remarkable for all that it accomplishes: reviewing experimental work on protein synthesis by biochemists, proposing the striking new ideas of the Sequence Hypothesis and the Central Dogma, reviewing previous work on the genetic code, and presenting an elegant possible coding scheme. The most important section of Crick's paper for our purposes is the section entitled "Ideas about Protein Synthesis.” There Crick said:

My own thinking (and that of many of my colleagues) is based on two general principles, which I call the Sequence Hypothesis and the Central Dogma.

$\cdots$

Biological Theory 1(3) 2006
The Sequence Hypothesis

... In its simplest form it assumes that the specificity of a piece of nucleic acid is expressed solely by the sequence of its bases, and that this sequence is a (simple) code for the amino acid sequence of a particular protein.

$\cdots$

\section{The Central Dogma}

This states that once 'information' has passed into protein it cannot get out again. In more detail, the transfer of information from nucleic acid to nucleic acid, or from nucleic acid to protein may be possible, but transfer from protein to protein, or from protein to nucleic acid, is impossible. Information means here the precise determination of sequence, either of bases in the nucleic acid or of amino acid residues in the protein. (Crick 1958: 152-153; italics in original, underlining added).

A key point to note here is that Crick's characterization of information is not that it passively carries the code for protein sequence. Instead, he said that information means the precise determination of sequence. Determination requires the operation of a mechanism. Following the flow of information in the mechanism of protein synthesis traces the flow through stages from DNA to what was later found to be messenger RNA (Jacob and Monod 1961) to protein.

Code and information and mechanism were all key concepts here. As Kay (2000) has shown, in the 1940s code and information were not so tightly coupled. Erwin Schrödinger, in his 1944 What is Life?, speculated about the "code-script carried by genes but did not adopt the term "information" (Kay 2000: 65-66). According to Kay, "Up until around 1950 molecular biologists ... described genetic mechanisms without ever using the term information" (Kay 2000: 328).

Sahotra Sarkar (1996) said that "information" was introduced into bacterial genetics in print in 1953 in a paper by the geneticist Boris Ephrussi, with coauthors James Watson, Urs Leopold, and J. J. Weigle. In that paper, they suggested the term "interbacterial information" for "sexual recombination in bacteria." They stressed that "information" did not imply transfer of material substances (Ephrussi et al. 1953: 701). In the most recent segment of his autobiography, Watson says that this was a "silly note about terminology in bacterial genetics," written as a "spoof of the turgid writings of Joshua Lederberg, justly much famed for his 1946 discovery at Yale University that bacteria genetically recombine" (Watson 2002: 15). Lederberg (personal communication 2002) said that the paper had no influence.

More importantly, Watson and Crick, in their second paper of 1953 that discussed the genetical implications of their recently discovered (Watson and Crick 1953a) double-helical structure of DNA, used both "code" and "information": "it therefore seems likely that the precise sequence of the bases 
is the code which carries the genetical information" (Watson and Crick 1953b: 244; italics added).

It is important to distinguish the genetic code from the information for determination of the sequence of a protein (Darden and Tabery 2005). The genetic code is the set of relations between three bases, called a "codon," and one amino acid. After the code was discovered experimentally by biochemists, first, and then also by geneticists, Crick provided a table to look up these coded relations (see, e.g., Crick 1988: 170 , or a molecular biology textbook). For example, CAC codes for histidine. Information, on the other hand, is specified by the linear order of the codons in nucleic acids that, with appropriate cellular machinery, determines the amino acid sequence of a protein. The number of codons differs for proteins of different lengths.

The philosopher Gregory Morgan wrote to Crick to inquire as to whether Shannon's information theory influenced him. In a letter of 1998, which Greg Morgan kindly showed me, Crick replied that he was not influenced at all by Shannon's actual theory of information. Instead, he said that what he had in mind was the Morse code and that he used "information" because it seemed the obvious term. Supporting this connection between the genetic code and the Morse code is a passage from Crick's 1988 book, What Mad Pursuit. In discussing problems with simplicity as a guide to theory construction in biology, Crick said:

Even so, there is a simplicity of a sort in the genetic code. The codons all have just three bases. The Morse code, by contrast, has symbols of different lengths, the shorter ones coding the more frequent letters. This allows the code to be more efficient, but such a property may have been too difficult for nature to evolve at that early time. (Crick 1988: 139)

We can conclude that "information" as used by Crick is a coded linear pattern that determines a corresponding pattern from one stage to the next in the mechanism of protein synthesis, given appropriate conditions.

\section{Mechanisms in Molecular Biology}

Determination of corresponding pattern requires the operation of a mechanism. A general discussion of mechanisms will provide a framework for analyzing the flow of information in molecular biological mechanisms.

A number of philosophers of science have noted the importance of mechanisms, including Wimsatt (1972), Brandon (1985), Burian (1996), and Thagard (1999). Mechanisms produce a phenomenon (Machamer, Darden, and Craver 2000), carry out a task (Bechtel and Richardson 1993), or exhibit a behavior (Glennan 1996). Peter Machamer, Carl Craver, and I characterize mechanisms as "entities and activities organized such that they are productive of regular changes from start or set-up to finish or termination conditions" (Machamer, Darden, and Craver 2000: 3).

Types of entities include ions, macromolecules (such as proteins and the nucleic acids, DNA and RNA), and cellular structures, such as ribosomes. Types of activities include geometrico-mechanical activities, such as lock and key docking of an enzyme and its substrate, and chemical bonding activities, such as strong covalent bonding and weak hydrogen bonding. To identify a mechanism, one first identifies a phenomenon produced by a mechanism, such as protein synthesis, and then one works to identify the entities, activities, and their organization in the mechanism that produces that phenomenon.

The entities and activities are organized in stages with productive continuity from beginning to end. That is, each stage gives rise to, allows, drives, or makes the next stage (Darden and Craver 2002). One goal in discovering a mechanism is to reveal the mechanism's productive continuity. Any breaks in our understanding of that productive continuity constitute incompleteness yet to be filled.

Mechanism schemas (Skipper 1999; Machamer, Darden, and Craver 2000) are abstract frameworks for mechanisms. They contain placeholders for the components of the mechanism, and they indicate, with variable degrees of abstraction, how the components are organized. These placeholders may characterize a component's role (Craver 2001) in the mechanism and show the context into which it must fit.

Many discoveries in biology involve discovering a mechanism schema. The general knowledge in molecular biology, for example, can be viewed as consisting of knowledge of a set of mechanism schemas. So far as I know, the phrase "theory of molecular biology" is not used. Instead, the general knowledge in the field is knowledge of a set of related mechanisms. For example, DNA $\rightarrow$ RNA $\rightarrow$ protein is a mechanism schema for the mechanism of protein synthesis. This diagram is James Watson's (e.g., 1965) version of the Central Dogma, a version not endorsed by Crick (1970). (For discussion of Watson's versus Crick's versions, see Keyes [1999a, 1999b].) Other schemas are found in molecular biology, such as the schema for DNA replication and the many schemas for regulation of gene expression. (A similar point about the importance of mechanisms in molecular biology was made in Burian [1996].)

Mechanism schemas play the roles usually attributed to theories: they abstractly encapsulate general knowledge; they have varying scopes of applicability; they may be instantiated to provide explanations or predictions of particular phenomena. When the schema is instantiated, it depicts a productively continuous mechanism from beginning to end, with no unfilled gaps.

In contrast, a mechanism sketch cannot (yet) be instantiated. Components are (as yet) unknown. Sketches may have 
black boxes for missing components whose function is not yet known. They may also have gray boxes, whose functional role (Craver 2001) is known or conjectured; however, what specific entities and activities carry out that function in the mechanism are (as yet) unknown. The goal in mechanism discovery is to convert black and gray boxes into glass boxes, to continue the metaphor. ${ }^{1}$ A schema consists of glass boxes; these are abstract placeholders for which instantiations can (now, on demand) be provided. Well-supported theories in biology are often represented by schemas and schematic diagrams. An instantiated schema shows details of how the mechanism operates in a specific instance to produce the phenomenon.

Sketches and schemas are often depicted in diagrams. In 1952, when Watson (1968) said that he put the diagram DNA $\rightarrow$ RNA $\rightarrow$ protein above his desk at Cambridge, that diagram represented a mechanism sketch for the flow of information from DNA to protein. In 1952, the activities to instantiate the arrows and the structures of DNA and RNA were unknown. By about 1970, in contrast, that diagram represented a schema that could be instantiated with the details of the transcription of DNA to messenger RNA via the activity of hydrogen bonding and the translation of messenger RNA into protein, with the aid of ribosomes and transfer RNA (see, e.g., Watson 1970).

All these general features of mechanisms apply equally to mechanisms in which information flows and in which it does not.

\section{Flow of Information}

Given this characterization of mechanisms, sketches, and schemas, we can recast Crick's definition of information more explicitly in terms of mechanisms. Recall, "Information means here the precise determination of sequence, either of bases in the nucleic acid or of amino acid residues in the protein." As the mechanism of transcription operates, an enzyme opens the weak hydrogen bonds of the double helix of DNA and facilitates the hydrogen bonding of complementary bases of RNA along a linear stretch of DNA. The order of the bases in DNA is the information that determines the sequence of the bases in RNA. The messenger RNA then moves to the ribosomes where the translation machinery decodes the information to order the amino acids of the protein.

No single bit of matter flows from beginning to end. No radioactive tracer, for example, could be added to the system to trace the movement of matter from DNA to RNA to protein. Biochemists did use $\mathrm{C}^{14}$ labeled amino acids to trace their incorporation into a polypeptide (Zamecnik 1953); however, such flow of matter did not implicate DNA in the mechanism. Flow of matter from beginning to end does not occur in the protein synthesis mechanism; flow of information does.

Although energy is consumed at each stage and is used in varying ways, one would not say that energy is flowing through the mechanism from beginning to end. ${ }^{2}$ Biochemists were tracing energy flow in the form of ATP and its role in activating amino acids and its use in the formation of the peptide bond (e.g., Hoagland 1955). (That work did turn up the puzzling finding that an RNA was attached to the activated amino acid; as a result of the interfield interactions with molecular biology, that RNA was found to be what we now call transfer RNA; recounted in Hoagland [1996].)

In contrast to matter or energy, what flows from beginning to end in the protein synthesis mechanism is information, that is, a coded linear pattern of DNA base sequence that determines RNA base sequence that determines amino acid sequence.

All mechanisms have productive continuity from one stage to the next. The entities and activities of one stage give rise to the next stage. However, few mechanisms have information flow through multiple stages of the mechanism. The flow of information in the protein synthesis mechanism places specific constraints on the specific kinds of entities and activities to instantiate this flow and to carry out the determination of protein sequence. An advantage of weak hydrogen bonds is that they can be quickly broken and formed as complementary bases line up during the flow of information from DNA to RNA. As the order of codons needs to be preserved, stronger covalent bonds hold the backbone of messenger RNA together and thus keep the codons in their corresponding linear pattern. Fairly elaborate machinery is used in the mechanism for translating the order of the codons in the messenger RNA into the corresponding linear order of the amino acids in the protein. Structured ribosomal particles orient the messenger RNA so that a codon is available to hydrogen bond quickly to its anticodon on transfer RNA. Stronger covalent bonds attach amino acids to their transfer RNAs, ensuring that the proper amino acid is transported to the ribosome and added to the peptide chain in the proper order. Also, covalent peptide bonds link amino acids in the finished protein, ensuring stability for its linear order. Our understanding of the mechanism is aided by attending to the properties of both its entities and activities and the mechanism constraints that they satisfy, as they instantiate information flow. (For more discussion of constraints on mechanisms, such as componency, rate, and orientation constraints, see Craver and Darden [2001].)

Another feature of this mechanism, in contrast to others, is that information flow is unidirectional. As Crick's statement of the Central Dogma stresses, information cannot flow backward from protein sequence to nucleic acid sequence. No machinery exists to reverse translate protein sequence into base sequence. In a message to his biographer Robert Olby (personal communication 1997), Crick said that the issue of inheritance of acquired characters was not an issue when he formulated the Central Dogma. He was concerned with the relations of protein synthesis and nucleic acid synthesis: he was denying that amino acid sequences played a role in the sequentialization 
of bases in nucleic acids. Others had speculated about nucleoproteins and possibly mutual relations between proteins and nucleic acids (Olby 1970, 1994); Crick, trained as a protein crystallographer, saw no way for a reverse code to be devised, given the stereochemical constraints of protein structure (Crick 1988). Sometime later the Central Dogma was taken to be a denial of the inheritance of adaptive acquired characters by denying some sort of flow of information from proteins back to DNA base sequence. ${ }^{3}$

The colinearity of DNA bases and amino acids in proteins was expected, given Crick's Sequence Hypothesis and Central Dogma. However, it should be noted that neither of those claims rule out breaks in that colinearity. Neither Crick nor anyone else anticipated the discovery of intervening sequences (introns) separating the coding regions of DNA in eucaryotes. As the historian of biology Michel Morange (1998: 206) noted: "This discovery of split genes hit the world of biology like a bombshell." Nonetheless, Crick's two claims were stated sufficiently abstractly so that they still applied. Although the continuous linear pattern in the sequence of the DNA bases is, in some cases, spliced and edited, it nonetheless (for the most part) determines the linear order of amino acids in proteins.

My view of these exceptions is that they were and are fruitful anomalies that play an important role in discovering new mechanism stages between DNA and protein. The strategy of complicating an oversimplification is a common way that science changes (Darden 1991). Anomalies from a mismatch of the expected colinear pattern have served and continue to serve as a directive to look for additional stages in the mechanism to account for the alteration of pattern. In reasoning to resolve an anomaly, one can forward chain or backward chain through the simple DNA $\rightarrow$ RNA $\rightarrow$ protein schema to find locations of additional stages to produce the anomalous pattern. (For more on the strategies of forward and backward chaining in mechanism discovery, see Darden [2002, 2006: ch. 12].)

Larry Holmes's (1991, 1993) account of Krebs's discovery of the stages of the Krebs cycle showed how difficult it was for Krebs to find all the stages. When a reaction in vitro could go from a set of reactants to a product, there was little guidance to tell him that, instead, two more stages were spliced into the mechanism in vivo. There is no flow of information in the Krebs cycle and thus Krebs had less guidance about what intermediate stages might be present.

Contrast Krebs's lack of guidance with the guidance for eucaryotic molecular biologists provided by the flow of information in the protein synthesis mechanism. When the amino acid sequence of a protein did not share a colinear DNA base sequence found contiguously in the genome, such a mismatch was an immediately recognizable anomaly. Intervening stages in the mechanism were sought to account for the anomaly; several such cases provide examples of anomaly driven scien- tific change (a common strategy discussed in Darden [1991, 1995, 1998, 2006]).

Today the colinearity, or lack thereof, of DNA sequences and protein sequences still provides a fruitful guide to search for intervening stages in the mechanism. The comparison of genomes and proteoms (all the proteins in a cell or an organism) will continue to yield new insights into the diverse mechanism stages between DNA sequence and protein sequence when the information, the colinear coded pattern, has been altered.

The question of exactly how to characterize the difference between mechanisms in which information flows and those in which it does not still remains unanswered. One can point to numerous differences between, say, the mechanism of protein synthesis and the Krebs cycle. Whether "determination of corresponding pattern from one stage to the next (and so on)" will suffice to distinguish the difference remains to be explored.

\section{Biochemistry versus Molecular Biology}

For some time I was puzzled about the best ways to conceptually characterize the differences between the fields of molecular biology and biochemistry in the 1950s and 1960s (e.g., Darden and Maull 1977). Both fields worked at roughly the same size level and often studied the same mechanisms. The mechanism of protein synthesis was studied by both biochemists and molecular biologists. One important difference is connected to concern with flow of genetic information from DNA. Molecular biologists used the concept of information flow; biochemists came to it as a result of their interfield interactions with molecular biologists.

Attending just to the historical work on the mechanism of protein synthesis prior to work on cracking the genetic code, we can see sharp contrasts between the molecular biologists and biochemists. They differed from each other in the techniques they used, in the parts of the mechanism they investigated, and in their attention to different aspects of the productive continuity in the mechanism. While biochemists, such as Paul Zamecnik and M. Hoagland, were homogenizing rat livers and tracing centrifuge fractions (ably recounted in Hans-Jörg Rheinberger's 1997 book on Zamecnik's work), some molecular biologists were crystallizing macromolecules and subjecting them to $\mathrm{x}$-ray analysis and yet others were doing genetic crosses with bacteria and the bacterial virus, bacteriophage. While biochemists were subjecting the protein end of the mechanism to chemical analysis, molecular biologists began with the genetic material of DNA. Most importantly for our purposes here, while molecular biologists traced the flow of information, the biochemists studied the flow of matter or energy in the mechanism. While molecular biologists questioned how the genetic information contained in the order of bases along the DNA double helix served to order the amino 
acids in proteins, biochemists investigated the energy requirements for binding free amino acids in the strong, covalent, peptide bonds of proteins.

Despite these numerous differences, fruitful interfield interactions between molecular biology and biochemistry served to integrate their findings. As work proceeded from both ends of the mechanism, it converged in the middle with the discovery of new entities (three types of RNA and activated amino acids) and the activities of these entities (discussed in more detail in Darden and Craver [2002]). The result was an understanding of the flow of matter and energy in some parts of the protein synthesis mechanism, as well as flow of information from beginning to end. Molecular biologists thus brought the question of the roles of nucleic acids in information flow to add to the biochemists' concerns with matter and energy flow in the incorporation of amino acids into polypeptides. One might perilously engage in contrary-to-fact historical speculation as to how long it would have taken biochemists to find the roles of nuclei acids in determining amino acid sequence without molecular biology; a good while, I suspect.

\section{Different Uses of "Information"}

Crick's usage of "information" (which was connected to, but different from, his use of "code") differs from "information" as characterized in formal information theory (which is characterized mathematically) and from "information" as used in cognitive neuroscience (which is connected to "representation" and "information processing"; see, e.g., Grush 2001 and Victor, this issue).

Sahotra Sarkar (1996) argued persuasively that "information" as used in Shannon's 1948 theory is inapplicable to the usage of information in molecular biology: "what should be regarded as biologically informative-functional sequencesare exactly those that have low information content" in Shannon's sense (Sarkar 1996: 862).

Also noteworthy is that the term "representation" is not used in molecular biology. Consider the question: Does it make sense to say that the sequence of the protein is "represented" in the sequence of bases? The answer, it seems to me, might be either yes or no. Certainly, Crick in 1958 and subsequent molecular biologists have not used the term "representation." Perhaps this points to something importantly different about the molecular biological and cognitive neuroscience uses of "information." Consider an oversimplified view of representation in which something in the environment is "represented" via a flow of information from that environment back to the organism, which then can use that representation as a basis for action in that environment.

In later life, Crick worked in the neurobiology of the visual system. He used "information" and "representation" in this cognitive neurobiological sense, with no mention of code:
The main function of the visual system is to build a representation inside our head of objects in the world outside us. It has to do this from the complex signals reaching the retinas of our eyes. Though these signals have much information implicit in them, the brain needs to process this information to obtain explicit representations of what interests it. Thus the photoreceptors in our eyes respond to the wavelength of the impinging light coming from an object. But what the brain is mainly interested in is the reflectivity (the color) of an object, and it can extract this information even under quite different conditions of illumination of that object.

The visual system has been evolved to detect those many aspects of the real world that, in evolution, have been important for survival, such as the recognition of food, predators, and possible mates. It is especially interested in moving objects. Evolution will latch onto any features that will give useful information. (Crick 1988: 154-155; my emphasis)

For this cognitive neurobiological case, information flows from an object to the visual system that builds a representation of that object. In contrast, no information flows from the threedimensional structure of a protein back into the DNA. The lack of such a reverse flow—even its impossibility—is the key idea in the central dogma. No mechanism exists for producing such a reverse flow. Because the three-dimensional structure is necessary for creating the active sites on proteins, one might have expected the codons for active sites to have some special status in the DNA. The DNA seems very blind to the use to which its information is put.

Such blindness seems unfortunate because such a reverse flow might allow DNA to monitor its successes and failures during an organism's life, learn from them, and improve. Some biologists continue to search for mechanisms to generate inheritance of adaptive acquired characters (e.g., Steele et al. 1998). But no such mechanisms have been found despite diligent search for them for over 200 years. (For one of the latest attempts, see Cairns et al. [1988], Foster [1999], discussed in Darden [2006: ch. 11].) Instead, functional sequences are merely highly conserved by natural selection operating crossgenerationally.

To sum up this point about different uses of "information": molecular biology's usage of "information" can be positioned in something of a midpoint. It is used with more content than "information" of formal information theory and is used with less content than "information" in cognitive neuroscience, which talks of "representations," "information processing," and "learning."

\section{Conclusion}

Information in molecular biology can be characterized as the determination of a corresponding linear coded pattern in stages of a mechanism. Mechanisms operate to determine corresponding patterns in linear nucleic acid base sequences 
and in linear amino acid sequences in proteins; information flow is unidirectional in the mechanism of protein synthesis. Anomalies in colinearity point to additional stages spliced into the mechanism in particular cases. Molecular biology, in the history of the discovery of the mechanism of protein synthesis, traced information flow, in contrast to biochemistry's work on the flow of matter and energy from amino acids to polypeptides. Tracing information flow required finding the roles of DNA and messenger RNA in the mechanism; molecular biologists, not biochemists, discovered those roles. The interfield interactions between molecular biologists and biochemists led to discoveries of all the components of the mechanism of protein synthesis, where flow of matter from beginning to end does not occur but flow of information does.

\section{Acknowledgments}

This work was supported by the U.S. National Science Foundation under grant SBR-9817942. I thank Werner Callebaut for the invitation to the KLI workshop on information, which inspired me to think more deeply about information flow in mechanisms, and to the workshop participants for comments. Work on mechanisms has been done in collaboration with Peter Machamer and Carl Craver. Thanks to Bob Olby for sharing his communication with Crick about inheritance of acquired characters and for other helpful comments; to Matt Barker, Jim Bogen, and Carl Craver for helpful comments on an earlier draft; to Greg Morgan for sharing his letter from Crick about the Morse code, and to him and others in the DC History and Philosophy of Biology discussion group for helpful ideas.

\section{Notes}

1. The metaphor of black, gray, and glass boxes is from Hanson (1963: 3738), who used in it to refer to somewhat different stages in theory discovery.

2. As an aside we can note that we might be interested in tracing energy flow if, for instance, we were discussing mechanisms (even actual machines) that convert mechanical energy to electrical energy. I was recently white water rafting on a stream that is used for electricity production; I felt some of that energy as we crashed over rocks; I could dramatically feel the effects of energy and matter flow. Of course, no flow of information was involved.

3. I do not know when that interpretation emerged; Judson (1996) incorrectly attributes it to Crick in 1958 .

\section{References}

Bechtel W, Richardson RC (1993) Discovering Complexity: Decomposition and Localization as Strategies in Scientific Research. Princeton, NJ: Princeton University Press.

Brandon RB (1985) Grene on mechanism and reductionism: More than just a side issue. In: PSA 1984, Vol. 2 (Asquith PD, Kitcher P, eds), 345-353. East Lansing, MI: Philosophy of Science Association.
Burian RM (1996) Underappreciated pathways toward molecular genetics as illustrated by Jean Brachet's cytochemical embryology. In: The Philosophy and History of Molecular Biology: New Perspectives (Sarkar S, ed), 67-85. Dordrecht: Kluwer.

Cairns J, Overbaugh J, Miller S (1988) The origin of mutants. Nature 335: 142-145.

Craver CF (2001) Role functions, mechanisms, and hierarchy. Philosophy of Science 68: 53-74.

Craver CF, Darden L (2001) Discovering mechanisms in neurobiology: The case of spatial memory. In: Theory and Method in the Neurosciences (Machamer P, Grush R, McLaughlin P, eds), 112-137. Pittsburgh, PA: University of Pittsburgh Press.

Crick F (1958) On protein synthesis. Symposium of the Society of Experimental Biology 12: 138-163.

Crick F (1970) Central dogma of molecular biology. Nature 227: 561-563.

Crick F (1988) What Mad Pursuit: A Personal View of Scientific Discovery. New York: Basic Books.

Darden L (1991) Theory Change in Science: Strategies From Mendelian Genetics. New York: Oxford University Press.

Darden L (1995) Exemplars, abstractions, and anomalies: Representations and theory change in Mendelian and molecular genetics. In: Concepts, Theories, and Rationality in the Biological Sciences (Lennox JG, Wolters G, eds), 137-158. Pittsburgh, PA: University of Pittsburgh Press.

Darden L (1998) Anomaly-driven theory redesign: Computational philosophy of science experiments. In: The Digital Phoenix: How Computers are Changing Philosophy (Bynum TW, Moor J, eds), 62-78. Oxford: Blackwell.

Darden L (2002) Strategies for discovering mechanisms: Schema instantiation, modular subassembly, forward/backward chaining. Philosophy of Science 69: S354-S365.

Darden L (2006) Reasoning in Biological Discoveries: Mechanisms, Interfield Relations, and Anomaly Resolution. New York: Cambridge University Press.

Darden L, Craver C (2002) Strategies in the interfield discovery of the mechanism of protein synthesis. Studies in History and Philosophy of Biological and Biomedical Sciences 33: 1-28.

Darden L, Maull N (1977) Interfield theories. Philosophy of Science 44: 4364.

Darden L, Tabery J (2005) Molecular biology. In: The Stanford Encyclopedia of Philosophy (Zalta EN, ed). http://plato.stanford.edu/entries/molecularbiology/

Ephrussi B, Leopold U, Watson JD, Weigle JJ (1953) Terminology in bacterial genetics. Nature 171: 701.

Foster PL (1999) Mechanisms of stationary phase mutation: A decade of adaptive mutation. Annual Review of Genetics 33: 57-88.

Glennan SS (1996) Mechanisms and the nature of causation. Erkenntnis 44: $49-71$.

Grush R (2001) The semantic challenge to computational neuroscience. In: Theory and Method in the Neurosciences (Machamer P, Grush R, McLaughlin P, eds), 155-172. Pittsburgh, PA: University of Pittsburgh Press.

Hanson NR (1963) The Concept of the Positron: A Philosophical Analysis. Cambridge: Cambridge University Press.

Hoagland MB (1955) An enzymic mechanism for amino acid activation in animal tissues. Biochimica et Biophysica Acta 16: 288-289.

Hoagland MB (1996) Biochemistry or molecular biology? The discovery of 'soluble RNA'. Trends in Biological Sciences Letters (TIBS) 21: 77-80.

Holmes FL (1991) Hans Krebs: The Formation of a Scientific Life 1900-1933. Vol. 1. New York: Oxford University Press.

Holmes FL (1993) Hans Krebs: Architect of Intermediary Metabolism. Vol. 2. New York: Oxford University Press. 
Jacob F, Monod J (1961) Genetic regulatory mechanisms in the synthesis of proteins. Journal of Molecular Biology 3: 318-356.

Judson HF (1996) The Eighth Day of Creation: The Makers of the Revolution in Biology. Expanded ed. Cold Spring Harbor, NY: Cold Spring Harbor Laboratory Press.

Kay LE (2000) Who Wrote the Book of Life? A History of the Genetic Code. Stanford, CA: Stanford University Press.

Keyes M (1999a) The prion challenge to the "central dogma" of molecular biology, 1965-1991: I. Prelude to prions. Studies in the History and Philosophy of Biological and Biomedical Sciences 30: 1-19.

Keyes M (1999b) The prion challenge to the "central dogma" of molecular biology, 1965-1991. II. The problem with prions. Studies in the History and Philosophy of Biological and Biomedical Sciences 30: 181-218.

Machamer P, Darden L, Craver C (2000) Thinking about mechanisms. Philosophy of Science 67: 1-25.

Morange M (1998) A History of Molecular Biology. Translated from French by Matthew Cobb. Cambridge, MA: Harvard University Press.

Olby R (1970) Francis Crick, DNA, and the central dogma. In: The Twentieth Century Sciences (Holton G, ed), 227-280. New York: Norton.

Olby R (1994) The Path to the Double Helix: The Discovery of DNA. Revised ed. Mineola, NY: Dover.

Rheinberger H-J (1997) Towards a History of Epistemic Things: Synthesizing Proteins in the Test Tube. Stanford, CA: Stanford University Press.

Sarkar S (1996) Decoding "coding"_-information and DNA. BioScience 46: 857-864.
Schrödinger E (1944) What Is Life? Cambridge: Cambridge University Press.

Skipper RA (1999) Selection and the extent of explanatory unification. Philosophy of Science 66: S196-S209.

Steele EJ, Lindley RA, Blanden RB (1998) Lamarck's Signature. Reading, MA: Perseus Books.

Thagard P (1999) How Scientists Explain Disease. Princeton, NJ: Princeton University Press.

Victor JD (2006). Approaches to information-theoretic analysis of neural activity. Biological Theory 1: 302-316.

Watson JD (1965) Molecular Biology of the Gene. New York: Benjamin.

Watson JD (1968) The Double Helix. New York: New American Library.

Watson JD (1970) Molecular Biology of the Gene. 2nd ed. New York: Benjamin.

Watson JD (2002) Genes, Girls, and Gamow. New York: Knopf.

Watson JD, Crick FHC (1953a) A structure for deoxyribose nucleic acid. Nature 171: 737-738.

Watson JD, Crick FHC (1953b) Genetical implications of the structure of deoxyribonucleic acid. Nature 171: 964-967. Reprinted in: The Double Helix, A Norton Critical Edition (Stent G, ed), 241-246. New York: Norton, 1980.

Wimsatt WC (1972) Complexity and organization. In: PSA 1972 (Schaffner KF, Cohen RS, eds), 67-86. Dordrecht: Reidel.

Zamecnik PC (1953) Incorporation of radioactivity from DL-leucine-1-C ${ }^{14}$ into proteins of rat liver homogenate. Federation Proceedings 12: 295. 